\title{
STABILITY OF F-STATIONARY MAPS OF A CLASS OF FUNCTIONALS RELATED TO CONFORMAL MAPS
}

\author{
Yingbo Han, Shuxiang Feng and Hong Pan
}

\begin{abstract}
In this paper, we study a generalized functional $\Phi_{F}$ related to the conformality of maps between Riemannian manifolds. We derive the first variation formula and the second variation formula of $\Phi_{F}$, then we study the stability of $F$-stationary map from or into the standard sphere. We also introduce the $F$-stress energy tensor associated to $\Phi_{F}$ which is naturally linked to conservation law.
\end{abstract}

\section{Introduction}

Let $\left(M^{m}, g\right)$ and $\left(N^{n}, h\right)$ be compact Riemannian manifolds without boundary. A smooth map $u$ from $M$ into $N$ is called a conformal map if there exists a positive function $\varphi$ on $M$ such that $u^{*} h=\varphi g$, where $u^{*} h$ denotes the pullback of the metric $h$ by $u$, i.e.

$$
u^{*} h(X, Y)=h(d u(X), d u(Y)) .
$$

Recently, N. Nakauchi in [8] introduced the following functional,

$$
\Phi(u)=\frac{1}{4} \int_{M}\left\|T_{u}\right\|^{2} d v_{g},
$$

(see $[6,9])$ where $T_{u}$ is the symmetric 2 -tensor defined by

$$
T_{u}=u^{*} h-\frac{1}{m}\|d u\|^{2} g
$$

and $\left\|T_{u}\right\|^{2},\|d u\|^{2}$ as

$$
\left\|T_{u}\right\|^{2}=\sum_{i, j} T_{u}\left(e_{i}, e_{j}\right)^{2}, \quad\|d u\|^{2}=\sum_{i} h\left(d u\left(e_{i}\right), d u\left(e_{i}\right)\right) .
$$

with respect to a local orthonormal frame $\left(e_{1}, \ldots, e_{m}\right)$ on $(M, g)$. They gave the first variation formula and the second variation formula for this func-

2010 Mathematics Subject Classification. 58E20; 53C43.

Key words and phrases. $F$-stationary map, $F$-stress-energy tensor, stable.

Received August 21, 2012; revised January 29, 2013. 
tional. They also gave a kind of the monotonicity formula and a Bochner type formula.

On the other hand, following Baird and Eells [2], Ara [1] introduced the $F$-harmonic maps, generalizing harmonic maps. Let $F:[0, \infty) \rightarrow[0, \infty)$ be a $C^{2}$ function such that $F(0)=0$ and $F^{\prime}(t)>0$ for $t \in[0, \infty)$. A smooth map $u: M \rightarrow N$ is said to be an $F$-harmonic map if it is a critical point of the following $F$-energy functional $E_{F}$ given by

$$
E_{F}(u)=\int_{M} F\left(\frac{\|d u\|^{2}}{2}\right) d v_{g}
$$

with respect to any compactly supported variation of $u$. After this, there are many geometers who studied $F$-harmonic map such as $[4,5,7]$.

In this paper, we generalize and unify the concept of critical point of the functional $\Phi$. For this, we define the functional $\Phi_{F}$ by

$$
\Phi_{F}(u)=\int_{M} F\left(\frac{\left\|T_{u}\right\|^{2}}{4}\right) d v_{g}
$$

which is $\Phi$ if $F(t)=t$. We call $u$ an $F$-stationary map for $\Phi_{F}(u)$, if

$$
\left.\frac{d}{d t} \Phi_{F}\left(u_{t}\right)\right|_{t=0}=0
$$

for any compactly supported variation $u_{t}: M \rightarrow N$ with $u_{0}=u$. We derive the first variation formula and the second variation formula of $\Phi_{F}$. We also prove that every stable $F$-stationary map form a compact manifold $M$ into $S^{n}$ is weakly conformal, provided that

$$
\int_{M^{m}}\left\|T_{u}\right\|^{2}\left\{F^{\prime \prime}\left(\frac{\left\|T_{u}\right\|^{2}}{4}\right)\left\|T_{u}\right\|^{2}+(4-n) F^{\prime}\left(\frac{\left\|T_{u}\right\|^{2}}{4}\right)\right\} d v_{g}<0 .
$$

or every stable $F$-stationary map from $S^{m}$ is weakly conformal, provided that

$$
\int_{S^{m}}\left\|T_{u}\right\|^{2}\left\{F^{\prime \prime}\left(\frac{\left\|T_{u}\right\|^{2}}{4}\right)\left\|T_{u}\right\|^{2}+(4-m) F^{\prime}\left(\frac{\left\|T_{u}\right\|^{2}}{4}\right)\right\} d v_{g}<0 .
$$

We also introduce the $F$-stress energy tensor associated to $\Phi_{F}$ which is naturally linked to conservation law.

The contents of this paper is as follows:

1. Introduction.

2. Preliminaries.

3. The first variation formula for $\Phi_{F}(u)$.

4. $F$-stress energy tensor

5. The second variation formula for $\Phi_{F}(u)$.

6. Stable maps into spheres.

7. Stable maps from spheres. 


\section{Preliminaries}

Let $\left(M^{m}, g\right)$ and $\left(N^{n}, h\right)$ be compact Riemannian manifolds without boundary and let $u$ be a smooth map from $M$ to $N$. We recall the following notions.

Definition 2.1. (i) A smooth map $u$ is weakly conformal if there exists a non-negative function $\varphi$ on $M$ such that

$$
u^{*} h=\varphi g
$$

where $u^{*} h$ denotes the pullback of the metric $h$ by $u$, i.e.

$$
u^{*} h(X, Y)=h(d u(X), d u(Y)) .
$$

(ii) A smooth map $u$ is conformal if there exists a positive function $\varphi$ on satisfy the equation (1).

The condition (1) is equivalent to

$$
u^{*} h=\frac{1}{m}\|d u\|^{2} g
$$

Since taking the trace of the both sides of (1) with respect to the metric $g$, we have $\varphi=\frac{1}{m}\|d u\|^{2}$. Then $u$ is conformal if and only if it satisfies (2) with the assumption $\|d u\| \neq 0$. Note that $u$ is weakly conformal if and only if for any point $x \in M, u$ is conformal at $x$, or $d u_{x}=0$.

In order to state our results, we also need the following Lemmas.

Lemma 2.2 [8]. (a) $T_{u}$ is symmetric, i.e. $T_{u}(X, Y)=T_{u}(Y, X)$.

(b) $u$ is weakly conformal if and only if $T_{u}=0$.

(c) $\left\|T_{u}\right\|^{2}=\left\|u^{*} h\right\|^{2}-\frac{1}{m}\|d u\|^{4}$.

(d) $T_{u}$ is trace-free, i.e.

$$
\text { Trace }_{g} T_{u}=\sum_{i, j} g\left(e_{i}, e_{j}\right) T_{u}\left(e_{i}, e_{j}\right)=0
$$

where $e_{i}$ denotes a local orthornormal frame on $M$.

(e) The trace of $T_{u}$ with respect to the pullback $u^{*} h$ is equal to the norm of $T_{u}$, i.e.

$$
\operatorname{Trace}_{u^{*} h} T_{u}=\sum_{i, j} h\left(d u\left(e_{i}\right), d u\left(e_{j}\right)\right) T_{u}\left(e_{i}, e_{j}\right)=\left\|T_{u}\right\|^{2}
$$


We define an $u^{-1} T N$-valued 1 -form $\sigma_{u}$ on $M$ by

$$
\sigma_{u}(X)=\sum_{j} T_{u}\left(X, e_{j}\right) d u\left(e_{j}\right)=\sum_{j} h\left(d u(X), d u\left(e_{j}\right)\right) d u\left(e_{j}\right)-\frac{1}{m}\|d u\|^{2} d u(X)
$$

for any vector field $X$ on $M$.

Lemma 2.3 .

$$
\sum_{i} h\left(d u\left(e_{i}\right), \sigma_{u}\left(e_{i}\right)\right)=\left\|T_{u}\right\|^{2}
$$

Proof.

$$
\begin{aligned}
\sum_{i} h\left(d u\left(e_{i}\right), \sigma_{u}\left(e_{i}\right)\right)= & \sum_{i, j} h\left(d u\left(e_{i}\right), d u\left(e_{j}\right)\right) h\left(d u\left(e_{i}\right), d u\left(e_{j}\right)\right) \\
& -\frac{1}{m}\|d u\|^{2} g\left(e_{i}, e_{j}\right) h\left(d u\left(e_{i}\right), d u\left(e_{j}\right)\right) \\
= & \left\|u^{*} h\right\|^{2}-\frac{1}{m}\|d u\|^{4}=\left\|T_{u}\right\|^{2}
\end{aligned}
$$

\section{The first variation formula for $\Phi_{F}(u)$}

Let $\nabla$ and ${ }^{N} \nabla$ always denote the Levi-Civita connections of $M$ and $N$ respectively. Let $\tilde{\nabla}$ be the induced connection on $u^{-1} T N$ defined by $\tilde{\nabla}_{X} W={ }^{N}$ $\nabla_{d u(X)} W$, where $X$ is a tangent vector of $M$ and $W$ is a section of $u^{-1} T N$. We choose a local orthonormal frame field $\left\{e_{i}\right\}$ on $M$. We define the $F$-tension field $\tau_{F}(u)$ of $u$ by

$$
\begin{aligned}
\tau_{F}(u) & =-\delta\left(F^{\prime}\left(\frac{\left\|T_{u}\right\|^{2}}{4}\right) \sigma_{u}\right) \\
& =F^{\prime}\left(\frac{\left\|T_{u}\right\|^{2}}{4}\right) \operatorname{div}_{g}\left(\sigma_{u}\right)+\sigma_{u}\left(\operatorname{grad}\left(F^{\prime}\left(\frac{\left\|T_{u}\right\|^{2}}{4}\right)\right)\right) .
\end{aligned}
$$

Under the notation above we have the following:

Lemma 3.1 (The first variation formula). Let $u: M \rightarrow N$ be a smooth map. Then

$$
\left.\frac{d}{d t} \Phi_{F}\left(u_{t}\right)\right|_{t=0}=-\int_{M} h\left(\tau_{F}(u), V\right) d v_{g}
$$

where $V=\left.\frac{d}{d t} u_{t}\right|_{t=0}$.

Proof. Let $\Psi:(-\varepsilon, \varepsilon) \times M \rightarrow N$ be defined by $\Psi(t, x)=u_{t}(x)$, where $(-\varepsilon, \varepsilon) \times M$ is equipped with the product metric. We extend the vector fields 
$\frac{\partial}{\partial t}$ on $(-\varepsilon, \varepsilon), X$ on $M$ naturally on $(-\varepsilon, \varepsilon) \times M$, and denote those also by $\frac{\partial}{\partial t}$, $X$. Then

$$
V=\left.d \Psi\left(\frac{\partial}{\partial t}\right)\right|_{t=0}
$$

We shall use the same notations $\nabla$ and $\tilde{\nabla}$ for the Levi-Civita connection on $(-\varepsilon, \varepsilon) \times M$ and the induced connection on $\Psi^{-1} T N$.

Now we compute

(7) $\frac{\partial}{\partial t} F\left(\frac{\left\|T_{u_{t}}\right\|^{2}}{4}\right)$

$$
\begin{aligned}
& =F^{\prime}\left(\frac{\left\|T_{u_{t}}\right\|^{2}}{4}\right) \frac{1}{4} \frac{\partial}{\partial t}\left\|T_{u_{t}}\right\|^{2} \\
& =\frac{1}{2} F^{\prime}\left(\frac{\left\|T_{u_{t}}\right\|^{2}}{4}\right) \sum_{i, j=1}^{m} \frac{\partial T_{u_{t}}\left(e_{i}, e_{j}\right)}{\partial t} T_{u_{t}}\left(e_{i}, e_{j}\right)
\end{aligned}
$$$$
=\frac{1}{2} F^{\prime}\left(\frac{\left\|T_{u_{t}}\right\|^{2}}{4}\right) \sum_{i, j=1}^{m}\left\{\frac{\partial}{\partial t} h\left(d u_{t}\left(e_{i}\right), d u_{t}\left(e_{j}\right)\right)-\frac{1}{m} \frac{\partial\left\|d u_{t}\right\|^{2}}{\partial t} g\left(e_{i}, e_{j}\right)\right\} T_{u_{t}}\left(e_{i}, e_{j}\right)
$$$$
=\frac{1}{2} F^{\prime}\left(\frac{\left\|T_{u_{t}}\right\|^{2}}{4}\right) \sum_{i, j=1}^{m} \frac{\partial}{\partial t} h\left(d u_{t}\left(e_{i}\right), d u_{t}\left(e_{j}\right)\right) T_{u_{t}}\left(e_{i}, e_{j}\right)
$$$$
=\frac{1}{2} F^{\prime}\left(\frac{\left\|T_{u_{t}}\right\|^{2}}{4}\right) \sum_{i, j=1}^{m} \frac{\partial}{\partial t} h\left(d \Psi\left(e_{i}\right), d \Psi\left(e_{j}\right)\right) T_{u_{t}}\left(e_{i}, e_{j}\right)
$$$$
=F^{\prime}\left(\frac{\left\|T_{u_{t}}\right\|^{2}}{4}\right) \sum_{i, j=1}^{m} h\left(\tilde{\nabla}_{\partial / \partial t} d \Psi\left(e_{i}\right), d \Psi\left(e_{j}\right)\right) T_{u_{t}}\left(e_{i}, e_{j}\right)
$$$$
=F^{\prime}\left(\frac{\left\|T_{u_{t}}\right\|^{2}}{4}\right) \sum_{i, j=1}^{m} h\left(\tilde{\nabla}_{e_{i}} d \Psi\left(\frac{\partial}{\partial t}\right), d \Psi\left(e_{j}\right)\right) T_{u_{t}}\left(e_{i}, e_{j}\right)
$$$$
=F^{\prime}\left(\frac{\left\|T_{u_{t}}\right\|^{2}}{4}\right) \sum_{i, j=1}^{m} h\left(\tilde{\nabla}_{e_{i}} d \Psi\left(\frac{\partial}{\partial t}\right), d u_{t}\left(e_{j}\right)\right) T_{u_{t}}\left(e_{i}, e_{j}\right)
$$$$
=F^{\prime}\left(\frac{\left\|T_{u_{t}}\right\|^{2}}{4}\right) \sum_{i=1}^{m} h\left(\tilde{\nabla}_{e_{i}} d \Psi\left(\frac{\partial}{\partial t}\right), \sigma_{u_{t}}\left(e_{i}\right)\right)
$$$$
=F^{\prime}\left(\frac{\left\|T_{u_{t}}\right\|^{2}}{4}\right) \sum_{i=1}^{m}\left[e_{i} h\left(d \Psi\left(\frac{\partial}{\partial t}\right), \sigma_{u_{t}}\left(e_{i}\right)\right)-h\left(d \Psi\left(\frac{\partial}{\partial t}\right), \tilde{\nabla}_{e_{i}} \sigma_{u_{t}}\left(e_{i}\right)\right)\right],
$$ 
where we use that

$$
\sum_{i, j=1}^{m} g\left(e_{i}, e_{j}\right) T_{u_{t}}\left(e_{i}, e_{j}\right)=0
$$

for the forth equality, and

$$
\tilde{\nabla}_{\partial / \partial t} d \Psi\left(e_{i}\right)-\tilde{\nabla}_{e_{i}} d \Psi\left(\frac{\partial}{\partial t}\right)=d \Psi\left[\frac{\partial}{\partial t}, e_{i}\right]=0
$$

for the seventh equality. Let $X_{t}$ be a compactly supported vector field on $M$ such that $g\left(X_{t}, Y\right)=h\left(d \Psi\left(\frac{\partial}{\partial t}\right), \sigma_{u_{t}}(Y)\right)$ for any vector field $Y$ on $M$. Then

(8) $\frac{\partial}{\partial t} F\left(\frac{\left\|T_{u_{t}}\right\|^{2}}{4}\right)=F^{\prime}\left(\frac{\left\|T_{u_{t}}\right\|^{2}}{4}\right) \sum_{i=1}^{m} e_{i} g\left(X_{t}, e_{i}\right)$

$$
\begin{aligned}
& -F^{\prime}\left(\frac{\left\|T_{u_{t}}\right\|^{2}}{4}\right) \sum_{i=1}^{m}\left[h\left(d \Psi\left(\frac{\partial}{\partial t}\right), \tilde{\nabla}_{e_{i}} \sigma_{u_{t}}\left(e_{i}\right)\right)\right] \\
= & F^{\prime}\left(\frac{\left\|T_{u_{t}}\right\|^{2}}{4}\right) \sum_{i=1}^{m}\left[g\left(\nabla_{e_{i}} X_{t}, e_{i}\right)+g\left(X_{t}, \nabla_{e_{i}} e_{i}\right)\right] \\
& -F^{\prime}\left(\frac{\left\|T_{u_{t}}\right\|^{2}}{4}\right) \sum_{i=1}^{m} h\left(d \Psi\left(\frac{\partial}{\partial t}\right), \tilde{\nabla}_{e_{i}} \sigma_{u_{t}}\left(e_{i}\right)\right) \\
= & F^{\prime}\left(\frac{\left\|T_{u_{t}}\right\|^{2}}{4}\right) d i v_{g}\left(X_{t}\right) \\
& -F^{\prime}\left(\frac{\left\|T_{u_{t}}\right\|^{2}}{4}\right) \sum_{i=1}^{m} h\left(d \Psi\left(\frac{\partial}{\partial t}\right), \tilde{\nabla}_{e_{i}} \sigma_{u_{t}}\left(e_{i}\right)-\sigma_{u_{t}}\left(\nabla_{e_{i}} e_{i}\right)\right) \\
= & \operatorname{div}\left(F^{\prime}\left(\frac{\left\|T_{u_{t}}\right\|^{2}}{4}\right) X_{t}\right)-g\left(X_{t}, g r a d\left(F^{\prime}\left(\frac{\left\|T_{u_{t}}\right\|^{2}}{4}\right)\right)\right) \\
& -F^{\prime}\left(\frac{\left\|T_{u_{t}}\right\|^{2}}{4}\right) \sum_{i=1}^{m} h\left(d \Psi\left(\frac{\partial}{\partial t}\right), \tilde{\nabla}_{e_{i}} \sigma_{u_{t}}\left(e_{i}\right)-\sigma_{u_{t}}\left(\nabla_{e_{i}} e_{i}\right)\right) \\
& +\sigma_{u_{t}}\left(g r a d\left(F^{\prime}\left(\frac{\left\|T_{u_{t}}\right\|^{2}}{4}\right)\right) .\right. \\
& \operatorname{div}\left(F^{\prime}\left(\frac{\left\|T_{u_{t}}\right\|^{2}}{4}\right) X_{t}\right)-h\left(d \Psi\left(\frac{\partial}{\partial t}\right), F^{\prime}\left(\frac{\left\|T_{u_{t}}\right\|^{2}}{4}\right) d i v_{g} \sigma_{u_{t}}\right) \\
& \left.(g)^{2}\right)
\end{aligned}
$$


By (8) and Green's theorem, we get

$$
\begin{aligned}
\left.\frac{d}{d t} \Phi_{F}\left(u_{t}\right)\right|_{t=0}= & \left.\int_{M} \frac{\partial}{\partial t} F\left(\frac{\left\|T_{u_{t}}\right\|^{2}}{4}\right)\right|_{t=0} d v_{g} \\
= & -\int_{M} h\left(d \Psi\left(\frac{\partial}{\partial t}\right), F^{\prime}\left(\frac{\left\|T_{u_{t}}\right\|^{2}}{4}\right) d i v_{g} \sigma_{u_{t}}\right) \\
& +\left.\sigma_{u_{t}}\left(\operatorname{grad}\left(F^{\prime}\left(\frac{\left\|T_{u_{t}}\right\|^{2}}{4}\right)\right)\right)\right|_{t=0} d v_{g} \\
= & -\int_{M} h\left(\tau_{F}(u), V\right) d v_{g} .
\end{aligned}
$$

The first variation formula allows us to define the notion of $F$-stationary for the functional $\Phi_{F}$.

Definition 3.2. A smooth map $u$ is called $F$-stationary map for the functional $\Phi_{F}$ if it is a solution of the Euler-Lagrange equation $\tau_{F}(u)=0$.

\section{4. $\quad F$-stress energy tensor}

Following Baird [3], for a smooth map $u:(M, g) \rightarrow(N, h)$, we associate a symmetric 2-tensor $S_{F}$ to the functional $\Phi_{F}$ called the $F$-stress energy tensor

$$
S_{F}(X, Y)=F\left(\frac{\left\|T_{u}\right\|^{2}}{4}\right) g(X, Y)-F^{\prime}\left(\frac{\left\|T_{u}\right\|^{2}}{4}\right) h\left(\sigma_{u}(X), d u(Y)\right),
$$

where $X, Y$ are vector fields on $M$.

Proposition 4.1. Let $u:(M, g) \rightarrow(N, h)$ be a smooth map and $S_{F}$ be the associated F-stress energy tensor, then for each vector field $X$ on $M$, we have

$$
\left(\operatorname{div} S_{F}\right)(X)=-h\left(\tau_{F}(u), d u(X)\right) .
$$

Proof. Let $\nabla$ and ${ }^{N} \nabla$ denote the Levi-Civita connections of $M$ and $N$, respectively. Let $\tilde{\nabla}$ be the induced connection on $u^{-1} T N$. We choose a local orthonormal frame field $\left\{e_{i}\right\}$ around a point $P$ on $M$ with $\left.\nabla_{e_{i}} e_{j}\right|_{P}=0$.

Let $X$ be a vector field on $M$. At $P$, we compute

$$
\begin{aligned}
\left(\operatorname{div} S_{F}\right)(X) & =\sum_{i=1}^{m}\left(\nabla_{e_{i}} S_{F}\right)\left(e_{i}, X\right) \\
& =\sum_{i=1}^{m}\left\{e_{i}\left(S_{F}\left(e_{i}, X\right)\right)-S_{F}\left(\nabla_{e_{i}} e_{i}, X\right)-S_{F}\left(e_{i}, \nabla_{e_{i}} X\right)\right\}
\end{aligned}
$$




$$
\begin{aligned}
& =\sum_{i=1}^{m}\left\{e_{i}\left(F\left(\frac{\left\|T_{u}\right\|^{2}}{4}\right) g\left(e_{i}, X\right)\right)-e_{i}\left(F^{\prime}\left(\frac{\left\|T_{u}\right\|^{2}}{4}\right) h\left(\sigma_{u}\left(e_{i}\right), d u(X)\right)\right)\right. \\
& \left.-F\left(\frac{\left\|T_{u}\right\|^{2}}{4}\right) g\left(e_{i}, \nabla_{e_{i}} X\right)+F^{\prime}\left(\frac{\left\|T_{u}\right\|^{2}}{4}\right) h\left(\sigma_{u}\left(e_{i}\right), d u\left(\nabla_{e_{i}} X\right)\right)\right\} \\
& =\sum_{i=1}^{m}\left\{e_{i}\left(F\left(\frac{\left\|T_{u}\right\|^{2}}{4}\right)\right) g\left(e_{i}, X\right)-e_{i}\left(F^{\prime}\left(\frac{\left\|T_{u}\right\|^{2}}{4}\right)\right) h\left(\sigma_{u}\left(e_{i}\right), d u(X)\right)\right. \\
& -F^{\prime}\left(\frac{\left\|T_{u}\right\|^{2}}{4}\right) h\left(\tilde{\nabla}_{e_{i}} \sigma_{u}\left(e_{i}\right), d u(X)\right)-F^{\prime}\left(\frac{\left\|T_{u}\right\|^{2}}{4}\right) h\left(\sigma_{u}\left(e_{i}\right), \tilde{\nabla}_{e_{i}} d u(X)\right) \\
& \left.+F^{\prime}\left(\frac{\left\|T_{u}\right\|^{2}}{4}\right) h\left(\sigma_{u}\left(e_{i}\right), d u\left(\nabla_{e_{i}} X\right)\right)\right\} \\
& =X\left(F\left(\frac{\left\|T_{u}\right\|^{2}}{4}\right)\right)-h\left(\sigma_{u}\left(\operatorname{grad} F^{\prime}\left(\frac{\left\|T_{u}\right\|^{2}}{4}\right)\right), d u(X)\right) \\
& -F^{\prime}\left(\frac{\left\|T_{u}\right\|^{2}}{4}\right) h\left(\operatorname{div} \sigma_{u}, d u(X)\right)-\sum_{i} F^{\prime}\left(\frac{\left\|T_{u}\right\|^{2}}{4}\right) h\left(\sigma_{u}\left(e_{i}\right),\left(\nabla_{e_{i}} d u\right)(X)\right) \\
& =-h\left(\tau_{F}(u), d u(X)\right)+F^{\prime}\left(\frac{\left\|T_{u}\right\|^{2}}{4}\right) X\left(\frac{\left\|T_{u}\right\|^{2}}{4}\right) \\
& -F^{\prime}\left(\frac{\left\|T_{u}\right\|^{2}}{4}\right) \sum_{i} h\left(\sigma_{u}\left(e_{i}\right),\left(\nabla_{e_{i}} d u\right)(X)\right) \\
& =-h\left(\tau_{F}(u), d u(X)\right)+F^{\prime}\left(\frac{\left\|T_{u}\right\|^{2}}{4}\right) \frac{1}{4} X\left(\left\|u^{*} h\right\|^{2}-\frac{1}{m}\|d u\|^{4}\right) \\
& -F^{\prime}\left(\frac{\left\|T_{u}\right\|^{2}}{4}\right) \sum_{i} h\left(\sigma_{u}\left(e_{i}\right),\left(\nabla_{e_{i}} d u\right)(X)\right) \\
& =-h\left(\tau_{F}(u), d u(X)\right)+F^{\prime}\left(\frac{\left\|T_{u}\right\|^{2}}{4}\right) \\
& \times\left(\sum_{i, j} h\left(\tilde{\nabla}_{X} d u\left(e_{i}\right), d u\left(e_{j}\right)\right) h\left(d u\left(e_{i}\right), d u\left(e_{j}\right)\right)-\frac{1}{m}\|d u\|^{2} \sum_{i} h\left(\tilde{\nabla}_{X} d u\left(e_{i}\right), d u\left(e_{i}\right)\right)\right) \\
& -F^{\prime}\left(\frac{\left\|T_{u}\right\|^{2}}{4}\right) \sum_{i} h\left(\sigma_{u}\left(e_{i}\right),\left(\nabla_{e_{i}} d u\right)(X)\right)
\end{aligned}
$$




$$
\begin{aligned}
= & -h\left(\tau_{F}(u), d u(X)\right)+F^{\prime}\left(\frac{\left\|T_{u}\right\|^{2}}{4}\right) \sum_{i} h \\
& \times\left(\tilde{\nabla}_{X} d u\left(e_{i}\right),\left[\sum_{j} h\left(d u\left(e_{i}\right), d u\left(e_{j}\right)\right) d u\left(e_{j}\right)-\frac{1}{m}\|d u\|^{2} d u\left(e_{i}\right)\right]\right) \\
& -F^{\prime}\left(\frac{\left\|T_{u}\right\|^{2}}{4}\right) \sum_{i} h\left(\sigma_{u}\left(e_{i}\right),\left(\nabla_{e_{i}} d u\right)(X)\right) \\
= & -h\left(\tau_{F}(u), d u(X)\right)+F^{\prime}\left(\frac{\left\|T_{u}\right\|^{2}}{4}\right) \sum_{i} h\left(\left(\nabla_{X} d u\right)\left(e_{i}\right), \sigma_{u}\left(e_{i}\right)\right) \\
& -F^{\prime}\left(\frac{\left\|T_{u}\right\|^{2}}{4}\right) \sum_{i} h\left(\sigma_{u}\left(e_{i}\right),\left(\nabla_{e_{i}} d u\right)(X)\right) .
\end{aligned}
$$

Since $\left(\nabla_{X} d u\right)\left(e_{i}\right)=\left(\nabla_{e_{i}} d u\right)(X)$, we obtain

$$
\left(\operatorname{div} S_{F}\right)(X)=-h\left(\tau_{F}(u), d u(X)\right) .
$$

From the above Proposition, we know that if $u: M \rightarrow N$ is an $F$-stationary map, we have

$$
\operatorname{div} S_{F}=0
$$

that is, $u$ satisfies the $\Phi_{F}$-conservation law.

Recall that for two 2-tensors $T_{1}, T_{2} \in \Gamma\left(T^{*} M \otimes T^{*} M\right)$, their inner product is defined as follows;

$$
\left\langle T_{1}, T_{2}\right\rangle=\sum_{i j} T\left(e_{i}, e_{j}\right) T_{2}\left(e_{i}, e_{j}\right)
$$

where $\left\{e_{i}\right\}$ is an orthonormal basis of with respect to $g$. For a vector field $X \in \Gamma(T M)$, we denote by $\theta_{X}$ is dual one form i.e. $\theta_{X}(Y)=g(X, Y)$. The covariant derivative of $\theta_{X}$ gives a 2 -tensor field $\nabla \theta_{X}$ :

$$
\left(\nabla \theta_{X}\right)(Y, Z)=\left(\nabla_{Z} \theta_{X}\right)(Y)=g\left(\nabla_{Z} X, Y\right) .
$$

If $X=\nabla \varphi$ is the gradient of some function $\varphi$ on $M$, then $\theta_{X}=d \varphi$ and $\nabla \theta_{X}=\operatorname{Hess} \varphi$.

Lemma 4.2 (CF. [3, 4]). Let $T$ be a symmetric (0,2)-type tensor field and let $X$ be a vector field, then

$$
\operatorname{div}\left(i_{X} T\right)=(\operatorname{div} T)(X)+\left\langle T, \nabla \theta_{X}\right\rangle=(\operatorname{div} T)(X)+\frac{1}{2}\left\langle T, L_{X} g\right\rangle .
$$


Let $D$ be any bounded domain of $M$ with $C^{1}$ boundary. By using the stokes' theorem, we immediately have the following integral formula:

$$
\int_{\partial D} T(X, v) d s_{g}=\int_{D}\left[\left\langle T, \frac{1}{2} L_{X} g\right\rangle+(\operatorname{div} T)(X)\right] d v_{g}
$$

where $v$ is the unit outward normal vector field along $\partial D$. By (11) and (15), we have

$$
\int_{\partial D} S_{F}(X, v) d s_{g}=\int_{D}\left\langle S_{F}, \frac{1}{2} L_{X} g\right\rangle d v_{g} .
$$

\section{The second variation formula for $\Phi_{F}(u)$}

In this section, we calculate the second variation of the functional $\Phi_{F}(u)$.

THEOREM 5.1 (The second variation formula). Let $u:(M, g) \rightarrow(N, h)$ be an $F$-stationary map. Let $u_{s, t}: M \rightarrow N(-\varepsilon<s, t<\varepsilon)$ be a compactly supported twoparameter variation such that $u_{0,0}=u$ and set $V=\left.\frac{\partial}{\partial t} u_{s, t}\right|_{s, t=0}, W=\left.\frac{\partial}{\partial s} u_{s, t}\right|_{s, t=0}$.

$$
\begin{aligned}
\left.\frac{\partial^{2}}{\partial s \partial t} \Phi_{F}\left(u_{s, t}\right)\right|_{s, t=0}= & \int_{M} F^{\prime \prime}\left(\frac{\left\|T_{u}\right\|^{2}}{4}\right)\left\langle\tilde{\nabla} V, \sigma_{u}\right\rangle\left\langle\tilde{\nabla} W, \sigma_{u}\right\rangle d v_{g} \\
& +\int_{M} F^{\prime}\left(\frac{\left\|T_{u}\right\|^{2}}{4}\right) \sum_{i, j=1}^{m} h\left(\tilde{\nabla}_{e_{i}} V, \tilde{\nabla}_{e_{j}} W\right) T_{u}\left(e_{i}, e_{j}\right) d v_{g} \\
& +\int_{M} F^{\prime}\left(\frac{\left\|T_{u}\right\|^{2}}{4}\right) \sum_{i, j=1}^{m} h\left(\tilde{\nabla}_{e_{i}} V, d u\left(e_{j}\right)\right) h\left(\tilde{\nabla}_{e_{i}} W, d u\left(e_{j}\right)\right) d v_{g} \\
& +\int_{M} F^{\prime}\left(\frac{\left\|T_{u}\right\|^{2}}{4}\right) \sum_{i, j=1}^{m} h\left(\tilde{\nabla}_{e_{i}} V, d u\left(e_{j}\right)\right) h\left(d u\left(e_{i}\right), \tilde{\nabla}_{e_{j}} W\right) d v_{g} \\
& -\frac{2}{m} \int_{M} F^{\prime}\left(\frac{\left\|T_{u}\right\|^{2}}{4}\right) \sum_{i, j=1}^{m} h\left(\tilde{\nabla}_{e_{i}} V, d u\left(e_{i}\right)\right) h\left(d u\left(e_{j}\right), \tilde{\nabla}_{e_{j}} W\right) d v_{g} \\
& +\int_{M} F^{\prime}\left(\frac{\left\|T_{u}\right\|^{2}}{4}\right) \sum_{i, j=1}^{m} h\left(R^{N}\left(V, d u\left(e_{i}\right)\right) W, d u\left(e_{j}\right)\right) T_{u}\left(e_{i}, e_{j}\right) d v_{g} .
\end{aligned}
$$

where $\langle$,$\rangle is the inner product on T^{*} M \otimes u^{-1} T N$ and $R^{N}$ is the curvature tensor of $N$.

We put

$$
I(V, W)=\left.\frac{\partial^{2}}{\partial s \partial t} \Phi_{F}\left(u_{s, t}\right)\right|_{s, t=0} .
$$


An $F$-stationary map $u$ is called stable if $I(V, V) \geq 0$ for any compactly supported vector field $V$ along $u$.

Proof. Let $\Psi:(-\varepsilon, \varepsilon) \times(-\varepsilon, \varepsilon) \times M \rightarrow N$ be defined by $\Phi(s, t, x)=u_{s, t}(x)$, where $(-\varepsilon, \varepsilon) \times(-\varepsilon, \varepsilon) \times M$ is equipped with the product metric. We extend the vector fields $\partial / \partial t$ on $(-\varepsilon, \varepsilon), \partial / \partial s$ on $(-\varepsilon, \varepsilon), X$ on $M$ naturally on $(-\varepsilon, \varepsilon) \times(-\varepsilon, \varepsilon) \times M$, and denote those also by $\partial / \partial t, \partial / \partial s$ and $X$. Then

$$
V=\left.d \Psi\left(\frac{\partial}{\partial t}\right)\right|_{s, t=0}, \quad W=\left.d \Psi\left(\frac{\partial}{\partial s}\right)\right|_{s, t=0} .
$$

We shall use the same notations $\nabla$ and $\tilde{\nabla}$ for the Levi-Civita connection on $(-\varepsilon, \varepsilon) \times(-\varepsilon, \varepsilon) \times M$ and the induced connection on $\Psi^{-1} T N$. We choose a local orthonormal frame $\left\{e_{i}\right\}_{i=1}^{m}$ around a point $P$ on $M$ with $\left.\nabla_{e_{i}} e_{j}\right|_{P}=0$.

Using (5) we have

$$
\begin{array}{r}
\left.\frac{\partial^{2}}{\partial s \partial t} \Phi_{F}\left(u_{s, t}\right)\right|_{s, t=0}=-\frac{\partial}{\partial s} \int_{M} h\left(d \Psi\left(\frac{\partial}{\partial t}\right), \sum_{i=1}^{m}\left\{\tilde{\nabla}_{e_{i}}\left(F^{\prime}\left(\frac{\left\|T_{u_{s, t}}\right\|^{2}}{4}\right) \sigma_{u_{s, t}}\left(e_{i}\right)\right)\right.\right. \\
\left.\left.-F^{\prime}\left(\frac{\left\|T_{u_{s, t}}\right\|^{2}}{4}\right) \sigma_{u_{s, t}}\left(\nabla_{e_{i}} e_{i}\right)\right\}\right)\left.\right|_{s, t=0} d v_{g} \\
=-\int_{M} h\left(d \Psi\left(\frac{\partial}{\partial t}\right), \sum_{i=1}^{m}\left\{\tilde{\nabla}_{\partial / \partial s} \tilde{\nabla}_{e_{i}}\left(F^{\prime}\left(\frac{\left\|T_{u_{s, t}}\right\|^{2}}{4}\right) \sigma_{u_{s, t}}\left(e_{i}\right)\right)\right.\right. \\
\left.\left.-\tilde{\nabla}_{\partial / \partial s}\left(F^{\prime}\left(\frac{\left\|T_{u_{s, t}}\right\|^{2}}{4}\right) \sigma_{u_{s, t}}\left(\nabla_{e_{i}} e_{i}\right)\right)\right\}\right)\left.\right|_{s, t=0} d v_{g}
\end{array}
$$

where we use the $F$-stationarity for the last equality. At $P$, we compute

$$
\begin{aligned}
h\left(d \Psi\left(\frac{\partial}{\partial t}\right), \sum_{i=1}^{m}\left\{\tilde{\nabla}_{\partial / \partial s} \tilde{\nabla}_{e_{i}}\left(F^{\prime}\left(\frac{\left\|T_{u_{s, t}}\right\|^{2}}{4}\right) \sigma_{u_{s, t}}\left(e_{i}\right)\right)\right.\right. \\
\left.\left.\quad-\tilde{\nabla}_{\partial / \partial s}\left(F^{\prime}\left(\frac{\left\|T_{u_{s, t}}\right\|^{2}}{4}\right) \sigma_{u_{s, t}}\left(\nabla_{e_{i}} e_{i}\right)\right)\right\}\right) \\
=h\left(d \Psi\left(\frac{\partial}{\partial t}\right), \sum_{i=1}^{m} \tilde{\nabla}_{e_{i}} \tilde{\nabla}_{\partial / \partial s}\left(F^{\prime}\left(\frac{\left\|T_{u_{s, t}}\right\|^{2}}{4}\right) \sigma_{u_{s, t}}\left(e_{i}\right)\right)\right) \\
+h\left(d \Psi\left(\frac{\partial}{\partial t}\right), \sum_{i=1}^{m} R^{N}\left(d \Psi\left(\frac{\partial}{\partial s}\right), d \Psi\left(e_{i}\right)\right)\left(F^{\prime}\left(\frac{\left\|T_{u_{s, t}}\right\|^{2}}{4}\right) \sigma_{u_{s, t}}\left(e_{i}\right)\right)\right)
\end{aligned}
$$

where we use $\left[\frac{\partial}{\partial s}, e_{i}\right]=0$. 
466

YINGBO HAN, SHUXIANG PENG AND HONG PAN

The first term in the right-hand side of (19) is

$$
\begin{aligned}
h(d \Psi & \left.\left(\frac{\partial}{\partial t}\right), \sum_{i=1}^{m} \tilde{\nabla}_{e_{i}} \tilde{\nabla}_{\partial / \partial s}\left(F^{\prime}\left(\frac{\left\|T_{u_{s, t}}\right\|^{2}}{4}\right) \sigma_{u_{s, t}}\left(e_{i}\right)\right)\right) \\
= & \sum_{i=1}^{m} e_{i} h\left(d \Psi\left(\frac{\partial}{\partial t}\right), \tilde{\nabla}_{\partial / \partial s}\left(F^{\prime}\left(\frac{\left\|T_{u_{s, t}}\right\|^{2}}{4}\right) \sigma_{u_{s, t}}\left(e_{i}\right)\right)\right) \\
& -\sum_{i=1}^{m} h\left(\tilde{\nabla}_{e_{i}} d \Psi\left(\frac{\partial}{\partial t}\right), \tilde{\nabla}_{\partial / \partial s}\left(F^{\prime}\left(\frac{\left\|T_{u_{s, t}}\right\|^{2}}{4}\right) \sigma_{u_{s, t}}\left(e_{i}\right)\right)\right)
\end{aligned}
$$

The second term in the right-hand side of (20) is

(21)

$$
\begin{aligned}
& \sum_{i=1}^{m} h\left(\tilde{\nabla}_{e_{i}} d \Psi\left(\frac{\partial}{\partial t}\right), \tilde{\nabla}_{\partial / \partial s}\left(F^{\prime}\left(\frac{\left\|T_{u_{s, t}}\right\|^{2}}{4}\right) \sigma_{u_{s, t}}\left(e_{i}\right)\right)\right. \\
&=\left[\sum_{i=1}^{m} h\left(\tilde{\nabla}_{e_{i}} d \Psi\left(\frac{\partial}{\partial t}\right), \sigma_{u_{s, t}}\left(e_{i}\right)\right)\right] F^{\prime \prime}\left(\frac{\left\|T_{u_{s, t}}\right\|^{2}}{4}\right) \frac{\partial}{\partial s}\left(\frac{\left\|T_{u_{s, t}}\right\|^{2}}{4}\right) \\
&+F^{\prime}\left(\frac{\left\|T_{u_{s, t}}\right\|^{2}}{4}\right) \sum_{i=1}^{m} h\left(\tilde{\nabla}_{e_{i}} d \Psi\left(\frac{\partial}{\partial t}\right), \tilde{\nabla}_{\partial / \partial s} \sigma_{u_{s, t}}\left(e_{i}\right)\right) \\
&=\left[\sum_{i=1}^{m} h\left(\tilde{\nabla}_{e_{i}} d \Psi\left(\frac{\partial}{\partial t}\right), \sigma_{u_{s, t}}\left(e_{i}\right)\right)\right] F^{\prime \prime}\left(\frac{\left\|T_{u_{s, t}}\right\|^{2}}{4}\right) \\
& {\left[\frac{1}{2} \sum_{i, j} \frac{\partial}{\partial s}\left[h\left(d \Psi\left(e_{i}\right), d \Psi\left(e_{j}\right)\right)-\frac{1}{m}\left\|d u_{u_{s}, t}\right\|^{2} g\left(e_{i}, e_{j}\right)\right] T_{u_{s, t}}\left(e_{i}, e_{j}\right)\right] } \\
&+F^{\prime}\left(\frac{\left\|T_{u_{s, t}}\right\|^{2}}{4}\right) \sum_{i=1}^{m} h\left(\tilde{\nabla}_{e_{i}} d \Psi\left(\frac{\partial}{\partial t}\right), \tilde{\nabla}_{\partial / \partial s} \sigma_{u_{s, t}}\left(e_{i}\right)\right) \\
&= {\left[\sum_{i=1}^{m} h\left(\tilde{\nabla}_{e_{i}} d \Psi\left(\frac{\partial}{\partial t}\right), \sigma_{u_{s, t}}\left(e_{i}\right)\right)\right] F^{\prime \prime}\left(\frac{\left\|T_{u_{s, t}}\right\|^{2}}{4}\right) } \\
& \times\left[\sum_{j=1}^{m} h\left(\tilde{\nabla}_{e_{j}} d \Psi\left(\frac{\partial}{\partial s}\right), \sigma_{u_{s, t}}\left(e_{j}\right)\right)\right] \\
&+F^{\prime}\left(\frac{\left\|T_{u_{s, t}}\right\|^{2}}{4}\right) \sum_{i=1}^{m} h\left(\tilde{\nabla}_{e_{i}} d \Psi\left(\frac{\partial}{\partial t}\right),\right.
\end{aligned}
$$




$$
\begin{aligned}
= & {\left[\sum_{i=1}^{m} h\left(\tilde{\nabla}_{e_{i}} d \Psi\left(\frac{\partial}{\partial t}\right), \sigma_{u_{s, t}}\left(e_{i}\right)\right)\right] F^{\prime \prime}\left(\frac{\left\|T_{u_{s, t}}\right\|^{2}}{4}\right)\left[\sum_{j=1}^{m} h\left(\tilde{\nabla}_{e_{j}} d \Psi\left(\frac{\partial}{\partial s}\right), \sigma_{u_{s, t}}\left(e_{j}\right)\right)\right] } \\
& +F^{\prime}\left(\frac{\left\|T_{u_{s, t}}\right\|^{2}}{4}\right) \sum_{i, j=1}^{m} h\left(\tilde{\nabla}_{e_{i}} d \Psi\left(\frac{\partial}{\partial t}\right), \tilde{\nabla}_{e_{j}} d \Psi\left(\frac{\partial}{\partial s}\right)\right) T_{u_{s, t}}\left(e_{i}, e_{j}\right) \\
& +F^{\prime}\left(\frac{\left\|T_{u_{s, t}}\right\|^{2}}{4}\right) \sum_{i, j=1}^{m} h\left(\tilde{\nabla}_{e_{i}} d \Psi\left(\frac{\partial}{\partial t}\right), d \Psi\left(e_{j}\right)\right) h\left(\tilde{\nabla}_{e_{i}} d \Psi\left(\frac{\partial}{\partial s}\right), d \Psi\left(e_{j}\right)\right) \\
& +F^{\prime}\left(\frac{\left\|T_{u_{s, t}}\right\|^{2}}{4}\right) \sum_{i, j=1}^{m} h\left(\tilde{\nabla}_{e_{i}} d \Psi\left(\frac{\partial}{\partial t}\right), d \Psi\left(e_{j}\right)\right) h\left(d \Psi\left(e_{i}\right), \tilde{\nabla}_{e_{j}} d \Psi\left(\frac{\partial}{\partial s}\right)\right) \\
& -F^{\prime}\left(\frac{\left\|T_{u_{s, t}}\right\|^{2}}{4}\right) \frac{2}{m} \sum_{i} h\left(\tilde{\nabla}_{e_{i}} d \Psi\left(\frac{\partial}{\partial t}\right), d \Psi\left(e_{i}\right)\right) \sum_{j} h\left(\tilde{\nabla}_{e_{j}} d \Psi\left(\frac{\partial}{\partial s}\right), d \Psi\left(e_{j}\right)\right),
\end{aligned}
$$

where we use that

$$
\sum_{i, j=1}^{m} g\left(e_{i}, e_{j}\right) T_{u_{s, t}}\left(e_{i}, e_{j}\right)=0
$$

for the third equality. Let $X_{1}, X_{2}, X_{3}, X_{4}$ and $X_{5}$ be compactly supported vector fields on $M$ such that

$$
\begin{aligned}
& g\left(X_{1}, Y\right)=F^{\prime \prime}\left(\frac{\left\|T_{u}\right\|^{2}}{4}\right)\left\langle\tilde{\nabla} W, \sigma_{u}\right\rangle h\left(\sigma_{u}(Y), V\right), \\
& g\left(X_{2}, Y\right)=F^{\prime}\left(\frac{\left\|T_{u}\right\|^{2}}{4}\right) \sum_{j=1}^{m} h\left(V, d u\left(e_{j}\right)\right) h\left(\tilde{\nabla}_{Y} W, d u\left(e_{j}\right)\right), \\
& g\left(X_{3}, Y\right)=F^{\prime}\left(\frac{\left\|T_{u}\right\|^{2}}{4}\right) \sum_{j=1}^{m} h\left(V, d u\left(e_{j}\right)\right) h\left(d u(Y), \tilde{\nabla}_{e_{j}} W\right), \\
& g\left(X_{4}, Y\right)=F^{\prime}\left(\frac{\left\|T_{u}\right\|^{2}}{4}\right) \sum_{j=1}^{m} h\left(V, \tilde{\nabla}_{e_{j}} W\right) T_{u}\left(Y, e_{j}\right), \\
& g\left(X_{5}, Y\right)=F^{\prime}\left(\frac{\left\|T_{u}\right\|^{2}}{4}\right) \sum_{j=1}^{m} h(V, d u(Y)) h\left(d u\left(e_{j}\right), \tilde{\nabla}_{e_{j}} W\right),
\end{aligned}
$$

for any vector field $Y$ on $M$, respectively. For the first term in the right-hand side of (20), we have 
468

YINGBO HAN, SHUXIANG FENG AND HONG PAN

(22)

$$
\begin{aligned}
& \sum_{i=1}^{m} e_{i} h\left(d \Psi\left(\frac{\partial}{\partial t}\right), \tilde{\nabla}_{\partial / \partial s}\left(F^{\prime}\left(\frac{\left\|T_{u_{s, t}}\right\|^{2}}{4}\right) \sigma_{u_{s, t}}\left(e_{i}\right)\right)\right) \\
& =\sum_{i=1}^{m} e_{i} h\left(F^{\prime \prime}\left(\frac{\left\|T_{u_{s, t}}\right\|^{2}}{4}\right)\left(\frac{1}{4} \frac{\partial}{\partial s}\left\|T_{u_{s, t}}\right\|^{2}\right) \sigma_{u_{s, t}}\left(e_{i}\right), d \Psi\left(\frac{\partial}{\partial t}\right)\right) \\
& +\sum_{i=1}^{m} e_{i} h\left(F^{\prime}\left(\frac{\left\|T_{u_{s, t}}\right\|^{2}}{4}\right) \tilde{\nabla}_{\partial / \partial s} \sigma_{u_{s, t}}\left(e_{i}\right), d \Psi\left(\frac{\partial}{\partial t}\right)\right) \\
& =\sum_{i=1}^{m} e_{i} h\left(F^{\prime \prime}\left(\frac{\left\|T_{u_{s, t}}\right\|^{2}}{4}\right)\left(\frac{1}{4} \frac{\partial}{\partial s}\left[\left\|u_{s, t}^{*} h\right\|^{2}-\frac{1}{m}\left\|d u_{s, t}\right\|^{4}\right]\right) \sigma_{u_{s, t}}\left(e_{i}\right), d \Psi\left(\frac{\partial}{\partial t}\right)\right) \\
& +\sum_{i=1}^{m} e_{i} h\left(F^{\prime}\left(\frac{\left\|T_{u_{s, t}}\right\|^{2}}{4}\right) \tilde{\nabla}_{\partial / \partial s} \sigma_{u_{s, t}}\left(e_{i}\right), d \Psi\left(\frac{\partial}{\partial t}\right)\right) \\
& =\sum_{i=1}^{m} e_{i}\left\{F^{\prime \prime}\left(\frac{\left\|T_{u_{s, t}}\right\|^{2}}{4}\right) \sum_{j=1}^{m} h\left(\sigma_{u_{s, t}}\left(e_{i}\right), d \Psi\left(\frac{\partial}{\partial t}\right)\right)\right. \\
& \left.\times h\left(\tilde{\nabla}_{e_{j}} d \Psi\left(\frac{\partial}{\partial s}\right), \sigma_{u_{s, t}}\left(e_{j}\right)\right)\right\} \\
& +\sum_{i=1}^{m} e_{i}\left\{F^{\prime}\left(\frac{\left\|T_{u_{s, t}}\right\|^{2}}{4}\right) \sum_{j=1}^{m} h\left(\tilde{\nabla}_{e_{i}} d \Psi\left(\frac{\partial}{\partial s}\right), d \Psi\left(e_{j}\right)\right)\right. \\
& \left.\times h\left(d \Psi\left(\frac{\partial}{\partial t}\right), d \Psi\left(e_{j}\right)\right)\right\} \\
& +\sum_{i=1}^{m} e_{i}\left\{F^{\prime}\left(\frac{\left\|T_{u_{s, t}}\right\|^{2}}{4}\right) \sum_{j=1}^{m} T_{u_{s, t}}\left(e_{i}, e_{j}\right) h\left(d \Psi\left(\frac{\partial}{\partial t}\right), \tilde{\nabla}_{e_{j}} d \Psi\left(\frac{\partial}{\partial s}\right)\right)\right\} \\
& +\sum_{i=1}^{m} e_{i}\left\{F^{\prime}\left(\frac{\left\|T_{u_{s, t}}\right\|^{2}}{4}\right) \sum_{j=1}^{m} h\left(d \Psi\left(e_{i}\right), \tilde{\nabla}_{e_{j}} d \Psi\left(\frac{\partial}{\partial s}\right)\right)\right. \\
& \left.\times h\left(d \Psi\left(\frac{\partial}{\partial t}\right), d \Psi\left(e_{j}\right)\right)\right\} \\
& -\frac{2}{m} \sum_{i=1}^{m} e_{i}\left\{F^{\prime}\left(\frac{\left\|T_{u_{s, t}}\right\|^{2}}{4}\right) \sum_{j=1}^{m} h\left(d \Psi\left(e_{j}\right), \tilde{\nabla}_{e_{j}} d \Psi\left(\frac{\partial}{\partial s}\right)\right)\right. \\
& \left.\times h\left(d \Psi\left(\frac{\partial}{\partial t}\right), d \Psi\left(e_{i}\right)\right)\right\},
\end{aligned}
$$


when $s=t=0$, (22) becomes

$$
\begin{aligned}
\sum_{i=1}^{m} e_{i} g & \left(X_{1}, e_{i}\right)+\sum_{i=1}^{m} e_{i} g\left(X_{2}, e_{i}\right)+\sum_{i=1}^{m} e_{i} g\left(X_{3}, e_{i}\right) \\
& +\sum_{i=1}^{m} e_{i} g\left(X_{4}, e_{i}\right)-\frac{2}{m} \sum_{i=1}^{m} e_{i} g\left(X_{5}, e_{i}\right) \\
= & \sum_{i=1}^{m} g\left(\nabla_{e_{i}} X_{1}, e_{i}\right)+\sum_{i=1}^{m} g\left(\nabla_{e_{i}} X_{2}, e_{i}\right)+\sum_{i=1}^{m} g\left(\nabla_{e_{i}} X_{3}, e_{i}\right) \\
& +\sum_{i=1}^{m} g\left(\nabla_{e_{i}} X_{4}, e_{i}\right)-\frac{2}{m} \sum_{i=1}^{m} g\left(\nabla_{e_{i}} X_{5}, e_{i}\right) \\
= & \operatorname{div}\left(X_{1}\right)+\operatorname{div}\left(X_{2}\right)+\operatorname{div}\left(X_{3}\right)+\operatorname{div}\left(X_{4}\right)-\frac{2}{m} \operatorname{div}\left(X_{5}\right) .
\end{aligned}
$$

By Green's theorem the integral of (23) vanishes. Theorem follows from (19)(23).

\section{Stable maps into spheres}

In this section we prove the following theorem

THeOREM 6.1. Let $u: M^{m} \rightarrow S^{n}$ be an F-stationary map from a compact Riemannian manifold $M$ into the $n$-dimensional standard sphere $S^{n}$. Assume that

$$
\int_{M^{m}}\left\|T_{u}\right\|^{2}\left\{F^{\prime \prime}\left(\frac{\left\|T_{u}\right\|^{2}}{4}\right)\left\|T_{u}\right\|^{2}+(4-n) F^{\prime}\left(\frac{\left\|T_{u}\right\|^{2}}{4}\right)\right\} d v_{g}<0 .
$$

Then $u$ is unstable.

Proof. In order to prove the instability of $u: M^{m} \rightarrow S^{n}$, we need to consider some special variational vector fields along $u$. To do this, choosing a local orthonormal frame field $\left\{\epsilon_{\alpha}\right\}, \alpha=1, \ldots, n$ around a point $P$ on $S^{n}$ with $\left.S^{n} \nabla_{\epsilon_{\alpha}} \epsilon_{\beta}\right|_{P}=0$ and choosing $\epsilon_{n+1}$ such that $\left\{\epsilon_{\alpha}, \epsilon_{n+1}\right\}$ is an orthonormal frame field of $R^{n+1}$. Meanwhile, taking a fixed orthonormal basis $E_{A}, A=1, \ldots, n+1$ of $R^{n+1}$ and setting

$$
V_{A}=\sum_{\alpha=1}^{n} v_{A}^{\alpha} \epsilon_{\alpha}, \quad v_{A}^{\alpha}=\left\langle E_{A}, \epsilon_{\alpha}\right\rangle, \quad v_{A}^{n+1}=\left\langle E_{A}, \epsilon_{n+1}\right\rangle,
$$

where $\langle$,$\rangle denotes the canonical Euclidean inner product. We shall consider the$ second variation 


$$
\begin{aligned}
I\left(V_{A}, V_{A}\right)= & \int_{M} F^{\prime \prime}\left(\frac{\left\|T_{u}\right\|^{2}}{4}\right)\left\langle\tilde{\nabla} V_{A}, \sigma_{u}\right\rangle\left\langle\tilde{\nabla}_{A}, \sigma_{u}\right\rangle d v_{g} \\
& +\int_{M} F^{\prime}\left(\frac{\left\|T_{u}\right\|^{2}}{4}\right) \sum_{i, j=1}^{m} h\left(\tilde{\nabla}_{e_{i}} V_{A}, \tilde{\nabla}_{e_{j}} V_{A}\right) T_{u}\left(e_{i}, e_{j}\right) d v_{g} \\
& +\int_{M} F^{\prime}\left(\frac{\left\|T_{u}\right\|^{2}}{4}\right) \sum_{i, j=1}^{m} h\left(\tilde{\nabla}_{e_{i}} V_{A}, d u\left(e_{j}\right)\right) h\left(\tilde{\nabla}_{e_{i}} V_{A}, d u\left(e_{j}\right)\right) d v_{g} \\
& +\int_{M} F^{\prime}\left(\frac{\left\|T_{u}\right\|^{2}}{4}\right) \sum_{i, j=1}^{m} h\left(\tilde{\nabla}_{e_{i}} V_{A}, d u\left(e_{j}\right)\right) h\left(d u\left(e_{i}\right), \tilde{\nabla}_{e_{j}} V_{A}\right) d v_{g} \\
& -\frac{2}{m} \int_{M} F^{\prime}\left(\frac{\left\|T_{u}\right\|^{2}}{4}\right) \sum_{i, j=1}^{m} h\left(\tilde{\nabla}_{e_{i}} V_{A}, d u\left(e_{i}\right)\right) h\left(d u\left(e_{j}\right), \tilde{\nabla}_{e_{j}} V_{A}\right) d v_{g} \\
& +\int_{M} F^{\prime}\left(\frac{\left\|T_{u}\right\|^{2}}{4}\right) \sum_{i} h\left(R^{S^{n}}\left(V_{A}, d u\left(e_{i}\right)\right) V_{A}, \sigma_{u}\left(e_{i}\right)\right) d v_{g} .
\end{aligned}
$$

At $P$, we compute

$$
\tilde{\nabla}_{e_{i}} V_{A}={ }^{S^{n}} \nabla_{d u\left(e_{i}\right)} V_{A}=-v_{A}^{n+1} d u\left(e_{i}\right) .
$$

From (25) and (27), we compute the following equations:

$$
\begin{aligned}
& F^{\prime \prime}\left(\frac{\left\|T_{u}\right\|^{2}}{4}\right) \sum_{A}\left\langle\tilde{\nabla} V_{A}, \sigma_{u}\right\rangle\left\langle\tilde{\nabla} V_{A}, \sigma_{u}\right\rangle \\
& =F^{\prime \prime}\left(\frac{\left\|T_{u}\right\|^{2}}{4}\right) \sum_{A, i, j} h\left(\tilde{\nabla}_{e_{i}} V_{A}, \sigma_{u}\left(e_{i}\right)\right) h\left(\tilde{\nabla}_{e_{j}} V_{A}, \sigma_{u}\left(e_{j}\right)\right) \\
& =F^{\prime \prime}\left(\frac{\left\|T_{u}\right\|^{2}}{4}\right) \sum_{A, i, j} v_{A}^{n+1} v_{A}^{n+1} h\left(d u\left(e_{i}\right), \sigma_{u}\left(e_{i}\right)\right) h\left(d u\left(e_{j}\right), \sigma_{u}\left(e_{j}\right)\right) \\
& =F^{\prime \prime}\left(\frac{\left\|T_{u}\right\|^{2}}{4}\right)\left\|T_{u}\right\|^{4}
\end{aligned}
$$

and

$$
\begin{aligned}
& F^{\prime}\left(\frac{\left\|T_{u}\right\|^{2}}{4}\right) \sum_{A} \sum_{i, j=1}^{m} h\left(\tilde{\nabla}_{e_{i}} V_{A}, \tilde{\nabla}_{e_{j}} V_{A}\right) T_{u}\left(e_{i}, e_{j}\right) \\
& =F^{\prime}\left(\frac{\left\|T_{u}\right\|^{2}}{4}\right) \sum_{i, j=1}^{m} \sum_{A} v_{A}^{n+1} v_{A}^{n+1} h\left(d u\left(e_{i}\right), d u\left(e_{j}\right)\right) T_{u}\left(e_{i}, e_{j}\right) \\
& =F^{\prime}\left(\frac{\left\|T_{u}\right\|^{2}}{4}\right)\left\|T_{u}\right\|^{2}
\end{aligned}
$$


and

$$
\begin{aligned}
& F^{\prime}\left(\frac{\left\|T_{u}\right\|^{2}}{4}\right) \sum_{A} \sum_{i, j=1}^{m} h\left(\tilde{\nabla}_{e_{i}} V_{A}, d u\left(e_{j}\right)\right) h\left(\tilde{\nabla}_{e_{i}} V_{A}, d u\left(e_{j}\right)\right) \\
& =F^{\prime}\left(\frac{\left\|T_{u}\right\|^{2}}{4}\right) \sum_{i, j=1}^{m} \sum_{A} v_{A}^{n+1} v_{A}^{n+1} h\left(d u\left(e_{i}\right), d u\left(e_{j}\right)\right) h\left(d u\left(e_{i}\right), d u\left(e_{j}\right)\right) \\
& =F^{\prime}\left(\frac{\left\|T_{u}\right\|^{2}}{4}\right)\left\|u^{*} h\right\|^{2}
\end{aligned}
$$

and

$$
\begin{aligned}
& F^{\prime}\left(\frac{\left\|T_{u}\right\|^{2}}{4}\right) \sum_{A} \sum_{i, j=1}^{m} h\left(\tilde{\nabla}_{e_{i}} V_{A}, d u\left(e_{j}\right)\right) h\left(d u\left(e_{i}\right), \tilde{\nabla}_{e_{j}} V_{A}\right) \\
& \quad=F^{\prime}\left(\frac{\left\|T_{u}\right\|^{2}}{4}\right) \sum_{i, j=1}^{m} \sum_{A} v_{A}^{n+1} v_{A}^{n+1} h\left(d u\left(e_{i}\right), d u\left(e_{j}\right)\right) h\left(d u\left(e_{i}\right), d u\left(e_{j}\right)\right) \\
& =F^{\prime}\left(\frac{\left\|T_{u}\right\|^{2}}{4}\right)\left\|u^{*} h\right\|^{2}
\end{aligned}
$$

and

$$
\begin{aligned}
& F^{\prime}\left(\frac{\left\|T_{u}\right\|^{2}}{4}\right) \sum_{A} \sum_{i, j=1}^{m} h\left(\tilde{\nabla}_{e_{i}} V_{A}, d u\left(e_{i}\right)\right) h\left(\tilde{\nabla}_{e_{j}} V_{A}, d u\left(e_{j}\right)\right) \\
& \quad=F^{\prime}\left(\frac{\left\|T_{u}\right\|^{2}}{4}\right) \sum_{i, j=1}^{m} \sum_{A} v_{A}^{n+1} v_{A}^{n+1} h\left(d u\left(e_{i}\right), d u\left(e_{i}\right)\right) h\left(d u\left(e_{j}\right), d u\left(e_{j}\right)\right) \\
& =F^{\prime}\left(\frac{\left\|T_{u}\right\|^{2}}{4}\right)\|d u\|^{4}
\end{aligned}
$$

and

$$
\begin{aligned}
& F^{\prime}\left(\frac{\left\|T_{u}\right\|^{2}}{4}\right) \sum_{i=1}^{m} \sum_{A} h\left(R^{S^{n}}\left(V_{A}, d u\left(e_{i}\right)\right) V_{A}, \sigma_{u}\left(e_{i}\right)\right) \\
& =F^{\prime}\left(\frac{\left\|T_{u}\right\|^{2}}{4}\right) \sum_{i=1}^{m} \sum_{A, \alpha, \beta} v_{A}^{\alpha} v_{A}^{\beta} h\left(R^{S^{n}}\left(\epsilon_{\alpha}, d u\left(e_{i}\right)\right) \epsilon_{\beta}, \sigma_{u}\left(e_{i}\right)\right) \\
& =F^{\prime}\left(\frac{\left\|T_{u}\right\|^{2}}{4}\right) \sum_{i=1}^{m} \sum_{\alpha} h\left(R^{S^{n}}\left(\epsilon_{\alpha}, d u\left(e_{i}\right)\right) \epsilon_{\alpha}, \sigma_{u}\left(e_{i}\right)\right)
\end{aligned}
$$




$$
\begin{aligned}
& =F^{\prime}\left(\frac{\left\|T_{u}\right\|^{2}}{4}\right) \sum_{i=1}^{m} \sum_{\alpha}\left[h\left(\epsilon_{\alpha}, \sigma_{u}\left(e_{i}\right)\right) h\left(\epsilon_{\alpha}, d u\left(e_{i}\right)\right)-h\left(d u\left(e_{i}\right), \sigma_{u}\left(e_{i}\right)\right) h\left(\epsilon_{\alpha}, \epsilon_{\alpha}\right)\right] \\
& =(1-n) F^{\prime}\left(\frac{\left\|T_{u}\right\|^{2}}{4}\right)\left\|T_{u}\right\|^{2} .
\end{aligned}
$$

From (26)-(33), we get

$$
\sum_{A=1}^{n+1} I\left(V_{A}, V_{A}\right)=\int_{M^{m}}\left\|T_{u}\right\|^{2}\left\{F^{\prime \prime}\left(\frac{\left\|T_{u}\right\|^{2}}{4}\right)\left\|T_{u}\right\|^{2}+(4-n) F^{\prime}\left(\frac{\left\|T_{u}\right\|^{2}}{4}\right)\right\} d v_{g}
$$

By (34) and the assumption, we have

$$
\sum_{A=1}^{n+1} I\left(V_{A}, V_{A}\right)<0
$$

and $u$ is unstable.

Corollary 6.2. Assume that (i) $F^{\prime \prime} \leq 0$ and $n \geq 5$, or (ii) $F^{\prime \prime}<0$ and $n=4$. Then any stable F-stationary map from a compact Riemannian manifold $M$ to $S^{n}$ is a weakly conformal map.

\section{Stable maps from spheres}

In this section we prove the following theorem

TheOrem 7.1. Let $u: S^{m} \rightarrow N$ be an F-stationary map. Assume that

$$
\int_{S^{m}}\left\|T_{u}\right\|^{2}\left\{F^{\prime \prime}\left(\frac{\left\|T_{u}\right\|^{2}}{4}\right)\left\|T_{u}\right\|^{2}+(4-m) F^{\prime}\left(\frac{\left\|T_{u}\right\|^{2}}{4}\right)\right\} d v_{g}<0 .
$$

Then $u$ is unstable.

Proof. In order to prove the instability of $u: S^{m} \rightarrow N$, we need to consider some special variational vector fields along $u$. To do this, choosing a local orthonormal frame field $\left\{e_{i}\right\}, i=1, \ldots, m$ around a point $P$ on $S^{m}$ with $\left.S^{m} \nabla_{e_{i}} e_{j}\right|_{P}=0$ and choosing $e_{m+1}$ such that $\left\{e_{i}, e_{m+1}\right\}$ is an orthonormal frame field of $R^{m+1}$. Meanwhile, taking a fixed orthonormal basis $E_{A}, A=1, \ldots, m+1$ of $R^{m+1}$ and setting

$$
V_{A}=\sum_{i=1}^{m} v_{A}^{i} e_{i}, \quad v_{A}^{i}=\left\langle E_{A}, e_{i}\right\rangle, \quad v_{A}^{m+1}=\left\langle E_{A}, e_{m+1}\right\rangle
$$

where $\langle$,$\rangle denotes the canonical Euclidean inner product. Then d u\left(V_{A}\right) \in$ $\Gamma\left(u^{-1} T N\right)$ and 


$$
\begin{gathered}
\sum_{A} v_{A}^{i} v_{A}^{j}=\sum_{A}\left\langle E_{A}, e_{i}\right\rangle\left\langle E_{A}, e_{j}\right\rangle=\delta_{i j}, \\
\nabla_{e_{i}} V_{A}=-v_{A}^{m+1} e_{i}, \\
\tilde{\nabla}_{e_{i}} d u\left(V_{A}\right)=-v_{A}^{m+1} d u\left(e_{i}\right)+v_{A}^{l} \tilde{\nabla}_{e_{i}} d u\left(e_{l}\right) .
\end{gathered}
$$

By using the condition $\tau_{F}(u)=-\delta\left(F^{\prime}\left(\frac{\left\|T_{u}\right\|^{2}}{4}\right) \sigma_{u}\right)=0$ and (38), we have

$$
\begin{aligned}
\int_{S^{m}} & \sum_{A=1}^{m+1} F^{\prime}\left(\frac{\left\|T_{u}\right\|^{2}}{4}\right)\left\langle(\triangle d u)\left(V_{A}\right), \sigma_{u}\left(V_{A}\right)\right\rangle d v_{g} \\
& =\int_{S^{m}} \sum_{A} v_{A}^{i} v_{A}^{j} F^{\prime}\left(\frac{\left\|T_{u}\right\|^{2}}{4}\right)\left\langle(\triangle d u)\left(e_{i}\right), \sigma_{u}\left(e_{j}\right)\right\rangle d v_{g} \\
& =\sum_{i} \int_{S^{m}} F^{\prime}\left(\frac{\left\|T_{u}\right\|^{2}}{4}\right)\left\langle(\triangle d u)\left(e_{i}\right), \sigma_{u}\left(e_{i}\right)\right\rangle \\
& =\int_{S^{m}} F^{\prime}\left(\frac{\left\|T_{u}\right\|^{2}}{4}\right)\left\langle(\triangle d u), \sigma_{u}\right\rangle \\
& =\int_{S^{m}}\left\langle\delta d u, \delta\left(F^{\prime}\left(\frac{\left\|T_{u}\right\|^{2}}{4}\right) \sigma_{u}\right)\right\rangle \\
& =0 .
\end{aligned}
$$

It follows from Weitzenböck formula that

(42) $-\sum_{k=1}^{m} R^{N}\left(d u(X), d u\left(e_{k}\right)\right) d u\left(e_{k}\right)+d u\left(\operatorname{Ric}^{S^{m}}(X)\right)=(\triangle d u)(X)+\left(\nabla^{2} d u\right)(X)$.

where $X$ is any smooth vector field on $S^{m}$ and $\left(\nabla^{2} d u\right)(X)=\sum_{i=1}^{m}\left[\nabla_{e_{i}} \nabla_{e_{i}} d u-\right.$ $\left.\nabla_{\nabla_{e_{i}} e_{i}} d u\right](X)$. With respect to the variational vector field $d u\left(V_{A}\right)$ along $u$, it follows form (41) and (42) that

$$
\begin{aligned}
\sum_{A} I\left(d u\left(V_{A}\right), d u\left(V_{A}\right)\right) \\
=\int_{M} F^{\prime \prime}\left(\frac{\left\|T_{u}\right\|^{2}}{4}\right) \sum_{A}\left\langle\tilde{\nabla} d u\left(V_{A}\right), \sigma_{u}\right\rangle^{2} d v_{g} \\
\quad+\int_{M} F^{\prime}\left(\frac{\left\|T_{u}\right\|^{2}}{4}\right) \sum_{i, j, A} h\left(\tilde{\nabla}_{e_{i}} d u\left(V_{A}\right), \tilde{\nabla}_{e_{j}} d u\left(V_{A}\right)\right) T_{u}\left(e_{i}, e_{j}\right) d v_{g} \\
\quad+\int_{M} F^{\prime}\left(\frac{\left\|T_{u}\right\|^{2}}{4}\right) \sum_{i, j, A} h\left(\tilde{\nabla}_{e_{i}} d u\left(V_{A}\right), d u\left(e_{j}\right)\right) h\left(\tilde{\nabla}_{e_{i}} d u\left(V_{A}\right), d u\left(e_{j}\right)\right) d v_{g}
\end{aligned}
$$




$$
\begin{aligned}
& +\int_{M} F^{\prime}\left(\frac{\left\|T_{u}\right\|^{2}}{4}\right) \sum_{i, j, A} h\left(\tilde{\nabla}_{e_{i}} d u\left(V_{A}\right), d u\left(e_{j}\right)\right) h\left(d u\left(e_{i}\right), \tilde{\nabla}_{e_{j}} d u\left(V_{A}\right)\right) d v_{g} \\
& -\frac{2}{m} \int_{M} F^{\prime}\left(\frac{\left\|T_{u}\right\|^{2}}{4}\right) \sum_{i, j, A} h\left(\tilde{\nabla}_{e_{i}} d u\left(V_{A}\right), d u\left(e_{i}\right)\right) h\left(d u\left(e_{j}\right), \tilde{\nabla}_{e_{j}} d u\left(V_{A}\right)\right) d v_{g} \\
& -\int_{M} F^{\prime}\left(\frac{\left\|T_{u}\right\|^{2}}{4}\right) \sum_{i} h\left(d u\left(\operatorname{Ric}^{S^{m}}\left(e_{i}\right)\right), \sigma_{u}\left(e_{i}\right)\right) d v_{g} \\
& \left.+\int_{M} F^{\prime}\left(\frac{\left\|T_{u}\right\|^{2}}{4}\right) \sum_{i} h\left(\left(\nabla^{2} d u\right)\left(e_{i}\right),\right) \sigma_{u}\left(e_{i}\right)\right) d v_{g} .
\end{aligned}
$$

At $P$, we compute

$$
\begin{aligned}
& F^{\prime \prime}\left(\frac{\left\|T_{u}\right\|^{2}}{4}\right) \sum_{A}\left\langle\tilde{\nabla} d u\left(V_{A}\right), \sigma_{u}\right\rangle^{2} \\
&=\sum_{A} F^{\prime \prime}\left(\frac{\left\|T_{u}\right\|^{2}}{4}\right)\left[\sum_{i}\left\langle\tilde{\nabla}_{e_{i}} d u\left(V_{A}\right), \sigma_{u}\left(e_{i}\right)\right\rangle\right]^{2} \\
&=F^{\prime \prime}\left(\frac{\left\|T_{u}\right\|^{2}}{4}\right) \sum_{A} \\
& \times {\left[\sum_{i}\left(-v_{A}^{m+1} h\left(d u\left(e_{i}\right), \sigma_{u}\left(e_{i}\right)\right)+v_{A}^{l} h\left(\tilde{\nabla}_{e_{i}} d u\left(e_{l}\right), \sigma_{u}\left(e_{i}\right)\right)\right)\right]^{2} } \\
&= F^{\prime \prime}\left(\frac{\left\|T_{u}\right\|^{2}}{4}\right) \sum_{A} v_{A}^{m+1} v_{A}^{m+1}\left[\sum_{i} h\left(d u\left(e_{i}\right), \sigma_{u}\left(e_{i}\right)\right)\right]^{2} \\
&-2 F^{\prime \prime}\left(\frac{\left\|T_{u}\right\|^{2}}{4}\right) \sum_{A} v_{A}^{m+1}\left[\sum_{i} h\left(d u\left(e_{i}\right), \sigma_{u}\left(e_{i}\right)\right)\right] \\
&\left.\times\left\{\sum_{l} v_{A}^{l}\left[\sum_{i} h\left(\tilde{\nabla}_{e_{i}} d u\left(e_{l}\right), \sigma_{u}\left(e_{i}\right)\right)\right)\right]\right\} \\
&+F^{\prime \prime}\left(\frac{\left\|T_{u}\right\|^{2}}{4}\right) \sum_{A}\left[\sum_{l} v_{A}^{l}\left[\sum_{i} h\left(\left(\nabla_{e_{i}} d u\right)\left(e_{l}\right), \sigma_{u}\left(e_{i}\right)\right)\right]\right]^{2} \\
&\left(\frac{T_{u} \|^{2}}{4}\right)\left[T_{u} \|^{4}+\sum_{l}\left[\sum_{i} h\left(\left(\nabla_{e_{l}} d u\right)\left(e_{i}\right), \sigma_{u}\left(e_{i}\right)\right)\right]^{2}\right]
\end{aligned}
$$

where we use the symmetry of $\nabla d u$ in the last equality. 
(45)

$$
\begin{aligned}
F^{\prime}\left(\frac{\left\|T_{u}\right\|^{2}}{4}\right) \sum_{i, j, A} h\left(\tilde{\nabla}_{e_{i}} d u\left(V_{A}\right), \tilde{\nabla}_{e_{j}} d u\left(V_{A}\right)\right) T_{u}\left(e_{i}, e_{j}\right) \\
=F^{\prime}\left(\frac{\left\|T_{u}\right\|^{2}}{4}\right) \sum_{A}\left[h \left(-v_{A}^{m+1} d u\left(e_{i}\right)+v_{A}^{k} \tilde{\nabla}_{e_{i}} d u\left(e_{k}\right),\right.\right. \\
\left.\left.\quad-v_{A}^{m+1} d u\left(e_{j}\right)+v_{A}^{l} \tilde{\nabla}_{e_{j}} d u\left(e_{l}\right)\right) T_{u}\left(e_{i}, e_{j}\right)\right] \\
=F^{\prime}\left(\frac{\left\|T_{u}\right\|^{2}}{4}\right)\left[\sum _ { A } v _ { A } ^ { k } v _ { A } ^ { l } h \left(\tilde { \nabla } _ { e _ { i } } d u \left(e^{2}-2 \sum_{A} v_{A}^{m+1} v_{A}^{k} h\left(d u\left(e_{i}\right), \tilde{\nabla}_{e_{j}} d u\left(e_{k}\right)\right) T_{u}\left(e_{i}, e_{j}\right)\right.\right.\right. \\
=F^{\prime}\left(\frac{\left\|T_{u}\right\|^{2}}{4}\right)\left[\left\|T_{u}\right\|^{2}+\sum_{i, j, k} h\left(\left(\nabla_{e_{k}} d u\right)\left(e_{i}\right),\left(\nabla_{e_{k}} d u\right)\left(e_{j}\right)\right) T_{u}\left(e_{i}, e_{j}\right)\right]
\end{aligned}
$$

and

$$
\begin{aligned}
F^{\prime}\left(\frac{\left\|T_{u}\right\|^{2}}{4}\right) \sum_{i, j, A} h\left(\tilde{\nabla}_{e_{i}} d u\left(V_{A}\right), d u\left(e_{j}\right)\right) h\left(\tilde{\nabla}_{e_{i}} d u\left(V_{A}\right), d u\left(e_{j}\right)\right) \\
=F^{\prime}\left(\frac{\left\|T_{u}\right\|^{2}}{4}\right) \sum_{A}\left[h\left(-v_{A}^{m+1} d u\left(e_{i}\right)+v_{A}^{k} \tilde{\nabla}_{e_{i}} d u\left(e_{k}\right), d u\left(e_{j}\right)\right)\right. \\
\left.\times h\left(-v_{A}^{m+1} d u\left(e_{i}\right)+v_{A}^{k} \tilde{\nabla}_{e_{i}} d u\left(e_{k}\right), d u\left(e_{j}\right)\right)\right] \\
=F^{\prime}\left(\frac{\left\|T_{u}\right\|^{2}}{4}\right)\left[\left\|u^{*} h\right\|^{2}+v_{A}^{k} v_{A}^{l} h\left(\tilde{\nabla}_{e_{i}} d u\left(e_{k}\right), d u\left(e_{j}\right)\right) h\left(\tilde{\nabla}_{e_{i}} d u\left(e_{l}\right), d u\left(e_{j}\right)\right.\right. \\
\left.-2 v_{A}^{m+1} v_{A}^{k} h\left(d u\left(e_{i}\right), d u\left(e_{j}\right)\right) h\left(\tilde{\nabla}_{e_{i}} d u\left(e_{k}\right), d u\left(e_{j}\right)\right)\right] \\
=F^{\prime}\left(\frac{\left\|T_{u}\right\|^{2}}{4}\right)\left[\left\|u^{*} h\right\|^{2}+\sum_{i, j, k} h\left(\left(\nabla_{e_{k}} d u\right)\left(e_{i}\right), d u\left(e_{j}\right)\right) h\left(\left(\nabla_{e_{k}} d u\right)\left(e_{i}\right), d u\left(e_{j}\right)\right]\right.
\end{aligned}
$$

and

$$
\begin{aligned}
& F^{\prime}\left(\frac{\left\|T_{u}\right\|^{2}}{4}\right) \sum_{i, j, A} h\left(\tilde{\nabla}_{e_{i}} d u\left(V_{A}\right), d u\left(e_{j}\right)\right) h\left(d u\left(e_{i}\right), \tilde{\nabla}_{e_{j}} d u\left(V_{A}\right)\right) \\
& \quad=F^{\prime}\left(\frac{\left\|T_{u}\right\|^{2}}{4}\right)\left[\left\|u^{*} h\right\|^{2}+\sum_{i, j, k} h\left(\left(\nabla_{e_{k}} d u\right)\left(e_{i}\right), d u\left(e_{j}\right)\right) h\left(d u\left(e_{i}\right),\left(\nabla_{e_{k}} d u\right)\left(e_{j}\right)\right]\right.
\end{aligned}
$$

and 


$$
\begin{aligned}
& F^{\prime}\left(\frac{\left\|T_{u}\right\|^{2}}{4}\right) \sum_{i, j, A} h\left(\tilde{\nabla}_{e_{i}} d u\left(V_{A}\right), d u\left(e_{i}\right)\right) h\left(d u\left(e_{j}\right), \tilde{\nabla}_{e_{j}} d u\left(V_{A}\right)\right) \\
& \quad=F^{\prime}\left(\frac{\left\|T_{u}\right\|^{2}}{4}\right)\left[\|d u\|^{4}+\sum_{i, j, k} h\left(\left(\nabla_{e_{k}} d u\right)\left(e_{i}\right), d u\left(e_{i}\right)\right) h\left(d u\left(e_{j}\right),\left(\nabla_{e_{k}} d u\right)\left(e_{j}\right)\right]\right.
\end{aligned}
$$

and

$$
\begin{aligned}
& F^{\prime}\left(\frac{\left\|T_{u}\right\|^{2}}{4}\right) \sum_{i} h\left(d u\left(\operatorname{Ric}^{S^{m}}\left(e_{i}\right)\right), \sigma_{u}\left(e_{i}\right)\right) \\
& \left.=(m-1) F^{\prime}\left(\frac{\left\|T_{u}\right\|^{2}}{4}\right) h\left(d u\left(e_{i}\right), \sigma_{u}\left(e_{i}\right)\right)\right) \\
& =(m-1) F^{\prime}\left(\frac{\left\|T_{u}\right\|^{2}}{4}\right)\left\|T_{u}\right\|^{2}
\end{aligned}
$$

and

$$
\begin{aligned}
F^{\prime}\left(\frac{\left\|T_{u}\right\|^{2}}{4}\right) \sum_{i} h\left(\left(\nabla^{2} d u\right)\left(e_{i}\right), \sigma_{u}\left(e_{i}\right)\right) \\
=F^{\prime}\left(\frac{\left\|T_{u}\right\|^{2}}{4}\right) \sum_{i, k} h\left(\tilde{\nabla}_{e_{k}} \tilde{\nabla}_{e_{k}} d u\left(e_{i}\right), \sigma_{u}\left(e_{i}\right)\right) \\
=e_{k}\left\{F^{\prime}\left(\frac{\left\|T_{u}\right\|^{2}}{4}\right) \sum_{i} h\left(\left(\nabla_{e_{k}} d u\right)\left(e_{i}\right), \sigma_{u}\left(e_{i}\right)\right)\right\} \\
-F^{\prime \prime}\left(\frac{\left\|T_{u}\right\|^{2}}{4}\right) \sum_{k}\left[\sum_{i} h\left(\left(\nabla_{e_{k}} d u\right)\left(e_{i}\right), \sigma_{u}\left(e_{i}\right)\right)\right]^{2} \\
\quad-F^{\prime}\left(\frac{\left\|T_{u}\right\|^{2}}{4}\right) \sum_{i, j, k} h\left(\left(\nabla_{e_{k}} d u\right)\left(e_{i}\right),\left(\nabla_{e_{k}} d u\right)\left(e_{j}\right)\right) T_{u}\left(e_{i}, e_{j}\right) \\
\quad-F^{\prime}\left(\frac{\left\|T_{u}\right\|^{2}}{4}\right) \sum_{i, j, k} h\left(\left(\nabla_{e_{k}} d u\right)\left(e_{i}\right), d u\left(e_{j}\right)\right) h\left(\left(\nabla_{e_{k}} d u\right)\left(e_{i}\right), d u\left(e_{j}\right)\right. \\
\quad-F^{\prime}\left(\frac{\left\|T_{u}\right\|^{2}}{4}\right) \sum_{i, j, k} h\left(\left(\nabla_{e_{k}} d u\right)\left(e_{i}\right), d u\left(e_{j}\right)\right) h\left(d u\left(e_{i}\right),\left(\nabla_{e_{k}} d u\right)\left(e_{j}\right)\right) \\
+\frac{2}{m} F^{\prime}\left(\frac{\left\|T_{u}\right\|^{2}}{4}\right) \sum_{i, j, k} h\left(\left(\nabla_{e_{k}} d u\right)\left(e_{i}\right), d u\left(e_{i}\right)\right) h\left(d u\left(e_{j}\right),\left(\nabla_{e_{k}} d u\right)\left(e_{j}\right)\right)
\end{aligned}
$$


By (43)-(50), we get

$$
\begin{aligned}
\sum_{A} I\left(d u\left(V_{A}\right), d u\left(V_{A}\right)\right) \\
\quad=\int_{S^{m}}\left\|T_{u}\right\|^{2}\left\{F^{\prime \prime}\left(\frac{\left\|T_{u}\right\|^{2}}{4}\right)\left\|T_{u}\right\|^{2}+(4-m) F^{\prime}\left(\frac{\left\|T_{u}\right\|^{2}}{4}\right)\right\} d v_{g} .
\end{aligned}
$$

By (51) and the assumption, we have

$$
\sum_{A} I\left(d u\left(V_{A}\right), d u\left(V_{A}\right)\right)<0
$$

and $u$ is unstable.

Corollary 7.2. Assume that (i) $F^{\prime \prime} \leq 0$ and $m \geq 5$, or (ii) $F^{\prime \prime}<0$ and $m=4$. Then any stable $F$-stationary map from $S^{m}$ is a weakly conformal map.

Acknowledgements. The authors would like to thank the referee whose valuable suggestions make this paper more perfect. Y. H. was supported by NSFC No. 10971029, No. 11201400, No. 11026062, Project of Henan Provincial Department of Education No. 2011A110015 and Talent youth teacher fund of Xinyang Normal University.

\section{REFERENCES}

[1] M. ArA, Geometry of $F$-harmonic maps, Kodai Math. J. 22 (1999), 243-263.

[2] P. Baird AND J. Eells, A conservation law for harmonic maps, Geometry Symposium Utrecht, 1980, Lecture notes in mathematics 894, Springer, Berlin, 1981, 1-25.

[ 3 ] P. BAIRD, Stess-energy tensors and the Linchnerowicz Laplacian, J. Geo. and Phys. 58 (2008), 1329-1342.

[ 4 ] Y. X. Dong AND S. S. WeI, On vanishing theorems for vector bundle valued $p$-forms and their applications, Comm. Math. Phys. 304 (2011), 329-368.

[5] Y. X. Dong, H. Z. Lin and G. L. Yang, Liouville theorems for $F$-harmonic maps and their applications, 2011, arXiv:1111.1882v1[math.DG].

[6] S. KAWAi AND N. NAKAuchi, Some result for stationary maps of a functional related to pullback metrics, Nonlinear analysis 74 (2011), 2284-2295.

[7] J. C. LiU, Liouville theorems of stable $F$-harmonic maps from compact convex hypersurfaces, Hiroshima Math. J. 36 (2006), 221-234.

[ 8 ] N. NaKaUCHI, A variational problem related to conformal maps, Osaka J. Math. 48 (2011), 719-741.

[9] N. Nakauchi and Y. Takenaka, A variational problem for pullback metrics, Ricerche Math. 60 (2011), 219-235. 
Yingbo Han

College of Mathematics and Information Science

Xinyang Normal University

XINYANG, 464000, HeNAN

P. R. CHINA

E-mail: yingbhan@yahoo.com.cn

Shuxiang Feng

College of Mathematics and Information Science

Xinyang Normal University

XinYang, 464000, Henan

P. R. CHINA

E-mail: fsxhyb@yahoo.com.cn

Hong Pan

College of Mathematics and Information Science Xinyang Normal University

Xinyang, 464000, Henan

P. R. CHINA

E-mail: panhong372@163.com 